\title{
Spatial coherence measurements of the EUV emission from laser-plasma source based on xenon/helium gas puff target
}

\author{
P. Wachulak ${ }^{1}$ A. Sarzyński ${ }^{1} \cdot$ A. Bartnik $^{1} \cdot$ T. $_{\text {Fok }}{ }^{1} \cdot$ L. Węgrzynski $^{1} \cdot$ J. Kostecki $^{1} \cdot$ \\ H. Fiedorowicz ${ }^{1}$
}

Received: 2 May 2017 / Accepted: 12 July 2017 / Published online: 19 July 2017

(C) The Author(s) 2017. This article is an open access publication

\begin{abstract}
In this paper, we present the first measurements of the partial spatial coherence of the EUV emission from xenon plasma in laser-plasma source, based on a double stream gas puff target. The Young double slit approach was employed to measure complex coherence factor of the EUV $\mathrm{Xe}$ emission at 13.5-nm wavelength in two orthogonal directions. The radius of coherence of $\sim 60 \mu \mathrm{m}$ was estimated at the distance of $2.1 \mathrm{~m}$ from the source. The number of coherently emitted photons was sufficient to demonstrate coherent imaging. Using partially coherent radiation from such source Gabor EUV holography was successfully demonstrated.
\end{abstract}

\section{Introduction}

X-ray nanoscience and nanotechnology often requires both spatially and temporary coherent sources of short wavelength radiation. Experiments and techniques, such as coherent diffraction imaging [1], holography [2] or interferometry [3], are not feasible without the use of coherent photon beams. The state-of-the-art sources of coherent radiation are synchrotrons and free electron lasers, where the pioneering experiments are being performed; however, in the recent years, more effort has been devoted to the development of more compact sources of such radiations, such as capillary discharge lasers [4], high-order harmonic generation sources [5], or laser-pumped solid-state EUV and SXR sources [6]. Those types of coherent EUV and SXR radiation were also

P. Wachulak

wachulak@gmail.com

1 Institute of Optoelectronics, Military University of Technology, 2 Kaliskiego Str., 00-908 Warsaw, Poland employed for important achievements in diffraction imaging [7], high-resolution holography [8], among others.

Among compact sources, there are also sources that use gas-type targets to produce laser-plasma-emitting short wavelength radiation. These sources are often referred to as sources based on gas jets [9]. Variations of those are double stream gas puff targets, which inject not one but two gasses into the interaction region. While the inner gas is called working gas, which is the material of the target, to which a specific elemental emission can be attributed, the other, outer gas surrounds the inner gas shaping its flow and increasing its density in the interaction region. Such source was already proven to be useful so far for various applications in metrology [10], full-field imaging [11], photoionization [12], polymer surface modification [13], radiobiology [14], etc. All those applications were related to the use of spatially incoherent EUV and SXR radiation; however, to this day, it was never used for coherent-type applications.

In this work, we would like to present the first to our knowledge attempt to obtain partially coherent EUV emission with usable photon flux from xenon/helium plasma, to perform coherent imaging experiments. We present the spatial coherence measurements, performed using Young double slit interferometry and demonstration of the use of such spatially coherent radiation to imaging. In the following chapters, the details about this work will be presented and discussed.

\section{Experimental setup}

The experimental setup for spatial coherence measurements of the emission from xenon plasma is depicted in Fig. 1a. An Nd:YAG laser beam, produced by NL 129 laser system (10 J/1-10 ns), from EKSPLA, Lithuania, having 


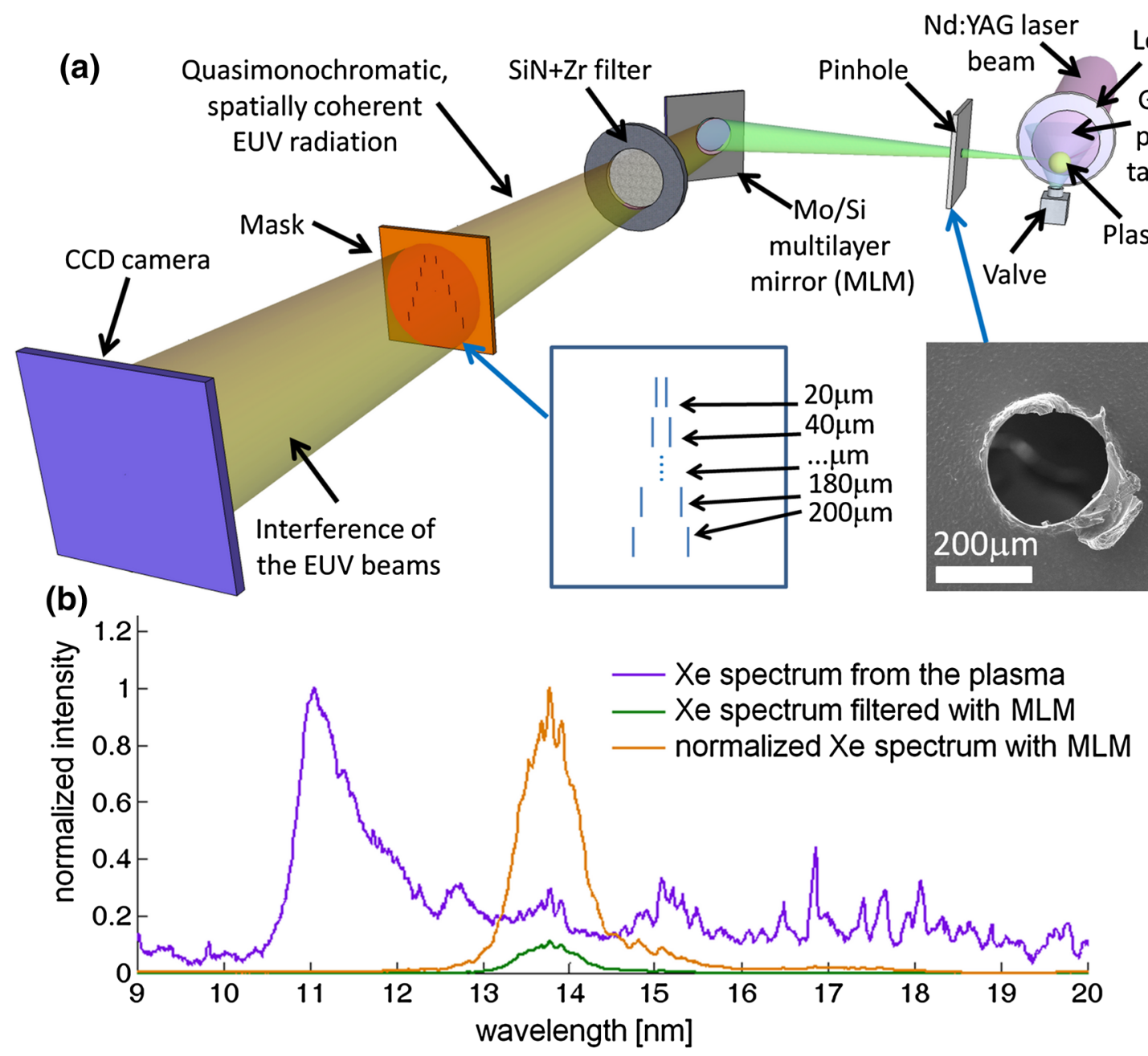

Fig. 1 Schematic description of the experimental setup for spatial coherence measurements based on Young double slit arrangement (a) and the emission spectrum from the Xe/He double stream gas puff target source (b)

pulse energy of $4.4 \mathrm{~J}$ and 4 ns time duration is focused by an $f=10 \mathrm{~cm}$ focal length lens onto a double stream gas puff target.

The target is formed by axisymmetric, collinear, stainless steel double nozzle, driven separately by two electromagnetic valves housed in a single body. The diameters of the nozzles are $0.4 \mathrm{~mm}$ for the inner nozzle and $0.7-1.5 \mathrm{~mm}$ for the outer, ring-shaped nozzle. The inner nozzle was supplied with a xenon gas at 8 bar backing pressure, while the outer nozzle was connected to helium pressurized to 6 bars. The double stream gas puff target was used due to increased target density, which is obtainable by shaping the flow of the inner gas into a vacuum through the use of the outer gas. In such case, higher inner gas density can be reached at certain distance away from the nozzle (in our case $1.5 \mathrm{~mm}$ ) to avoid degradation of the nozzle by a repeatable plasma formation. The valves were driven separately by a two-channel dedicated controller capable of independent adjustment of the delay and opening time for each valve in respect to the synchronization pulse arriving $1 \mathrm{~ms}$ before the laser pulse from the laser power supply. The delay time and opening times for each valve are 200/750 $\mu \mathrm{s}$ (Xe) and 400/550 $\mu \mathrm{s}$ (He), respectively. More details about the timing and valve synchronization can be found in [15]. Due to the interaction of the laser pulses with gaseous target, a laser-produced plasma is formed. Such plasma emits radiation in the broad range of wavelengths, from soft X-rays to infrared, depending on the gas used as a target, laser beam properties, and parameters of the focusing system. In this experiment, an efficient extreme ultraviolet emission was achieved from xenon gas, as already reported in [16].

In our system, plasma is not confined in any way and is allowed to expand freely in the tens of nanosecond time scale. This results in Gaussian-type plasma intensity distribution, as already reported multiple times, i.e., [17], with the size in the extreme ultraviolet spectral region to be $\sim 1 \mathrm{~mm}$ 
in diameter, so it can be hardly considered as a point source for applications requiring increased spatial coherence for the experiment. Thus, to improve the spatial coherence of the EUV beam by reducing the apparent source size, $0.25 \mathrm{~mm}$ in diameter pinhole, made by drilling a $100-\mu \mathrm{m}$-thick steel plate, was introduced $10 \mathrm{~mm}$ away from the plasma. This approach, in which high spatial coherence is achieved at the expense of photon flux by spatial filtering, is also very often employed at synchrotron facilities [18]. Smaller distance from the pinhole to the plasma caused the thermal damage to the pinhole induced by the plasma. The SEM micrograph of the pinhole, shown in Fig. 1a, shows that it is more like elliptical in shape with major and minor axes in horizontal and vertical directions, respectively. The major axis $D_{\mathrm{H}}$ was measured to be $251.5 \mu \mathrm{m}$ and the minor one $D_{\mathrm{V}}=234.2 \mu \mathrm{m}$. To monochromatize the EUV beam emitted from the xenon plasma, an Mo/Si multilayer mirror (MLM) was used. A $20 \times 20 \mathrm{~mm}^{2}$ in size flat mirror made by depositing $\mathrm{Mo} /$ Si multilayers on top of an Si wafer (Jena, Germany) has a peak reflectivity of $37 \%$ for an unpolarized beam at 13.5 $\mathrm{nm}$ wavelength, with \pm 0.5 - $\mathrm{nm}$ bandwidth and operated at an incidence angle of $45^{\circ}$. A typical spectral emission of the Xe plasma radiation reflected from such mirror in the extreme ultraviolet range from 9- to 20 -nm wavelength is depicted in Fig. 1b). To remove the visible light, which is also reflected efficiently from MLM, a combined thin-film filter was employed. A 200 -nm-thick silicon nitride membrane $5 \times 5 \mathrm{~mm}^{2}$ in size was coated with an additional layer of 200-nm-thick zirconium. Downstreaming the filter a set of slit pairs was located, at the distance of $2.1 \mathrm{~m}$ away from the plasma. Those slits were used in Young double slit experiment to produce an interference pattern in the far-field region. A set of ten slit pairs was fabricated in a $25-\mu \mathrm{m}$-thick copper foil by repetitive ablation of the material thorough its interactions with a focused laser beam. For that pulses from Nd:YAG laser (PL2210/SH/TH/FH from EKSPLA, Lithuania), operating at the third harmonic $\lambda=355 \mathrm{~nm}$, with a pulse energy of $20 \mu \mathrm{J}$, and time duration of $60 \mathrm{ps}$ at the repetition rate of $1 \mathrm{kHz}$ were used. The system was also equipped with an electromechanical shutter, SC-10 from Thorlabs, and galvanometric scanner, SS-IIE-10 [TY] from RAYLASE, Germany. A 50 passes at the scanning velocity of $2 \mathrm{~mm} / \mathrm{s}$ were sufficient to fabricate the slit arrangement used in this experiment. The initial separation of the slits should start from 20 to $200 \mu \mathrm{m}$ with a step of $20 \mu \mathrm{m}$; however, after fabrication, their separation was verified with the SEM measurements and the statistically measured values (from ten independent measurements) are depicted in Fig. 3. A typical slit pair, imaged with the SEM microscope, is shown in Fig. 2. It is for the slit separation of $\sim 40 \mu \mathrm{m}$. The quality of the fabricated slits is satisfactory. In addition, slit widths were also measured. Unfortunately, there is some discrepancy between the slit widths, as can be seen in the figure, of the order of $10 \%$ for min-max deviation of the slit widths. The length of each pair was $0.5 \mathrm{~mm}$ and separation between each slit pair was $0.2 \mathrm{~mm}$. The mask containing all slit pairs was $6.8 \times 0.2 \mathrm{~mm}^{2}$ in size. The mask was entirely illuminated by the light emitted from xenon plasma and filtered spatially by the pinhole and spectrally by MLM and set of filters. The fringe pattern is formed in the far field, where a CCD camera (Andor, DO934N-BN) was placed, $54 \mathrm{~cm}$ downstream of the mask. The camera has a chip with $1024 \times 1024$ pixels, each $13 \times 13 \mu \mathrm{m}^{2}$ in size. During the experiments, the chip was cooled down to $-20{ }^{\circ} \mathrm{C}$ to reduce its internal noise and the background.

\section{Results}

A two-slit (two pinhole) experiment had been used multiple times in measuring the spatial coherence of both X-ray lasers [19], HHG sources [20], and the capillary discharge laser [21]. If the beam is fully spatially coherent and its transversal coherence length $\rightarrow \infty$, which is in the case of a point source and flat wavefront, all the slits will have the highest possible modulation. The modulation is the difference between maximum $I_{\max }$ and minimum $I_{\min }$ intensity in the fringe interference pattern. For partially spatially coherent beam depending on the slit separation, the modulation and the period of that interference pattern will change [22]. If the slit separation increases, the modulation decreases and so-called visibility decreases as well. The visibility $V$ is the modulation normalized to the sum of minimum and maximum intensities in the interference region, as expressed in

$V=\frac{I_{\max }-I_{\min }}{I_{\max }+I_{\min }}$

An example of the interference patterns registered using the CCD camera behind the mask in the horizontal direction is presented in Fig. 3 for various slit separations.

The interferograms were time integrated over $50 \mathrm{EUV}$ pulses at $10 \mathrm{~Hz}$ source repetition rate. The figure shows also the intensity profiles (a) measured for each slit pair by averaging of ten line profiles in the CCD camera image (b). As can be observed, some residual modulation is visible up to a slit separation of $\sim 134 \mu \mathrm{m}$, corresponding to the visibility of $6 \%$. Beyond that point, the modulation reaches the noise level, which in this case corresponds to the visibility of $\sim 4 \%$, as indicated in Fig. 5 with a dashed-dotted line. The noise level means that the visibility smaller than this level cannot be measured simply because the CCD noise, present in the images, prohibits that. Due to the fact that our pinhole was not ideally circular, the interference measurements were carried out also in the direction perpendicular to the previous case. The results are presented in Fig. 4. Small change in 
Fig. 2 SEM micrograph of one pair of the slits with average separation of 36.6 cut in $20-\mu \mathrm{m}$-thick copper foil with a focused laser beam. Figure indicates also two measurements of the slit widths

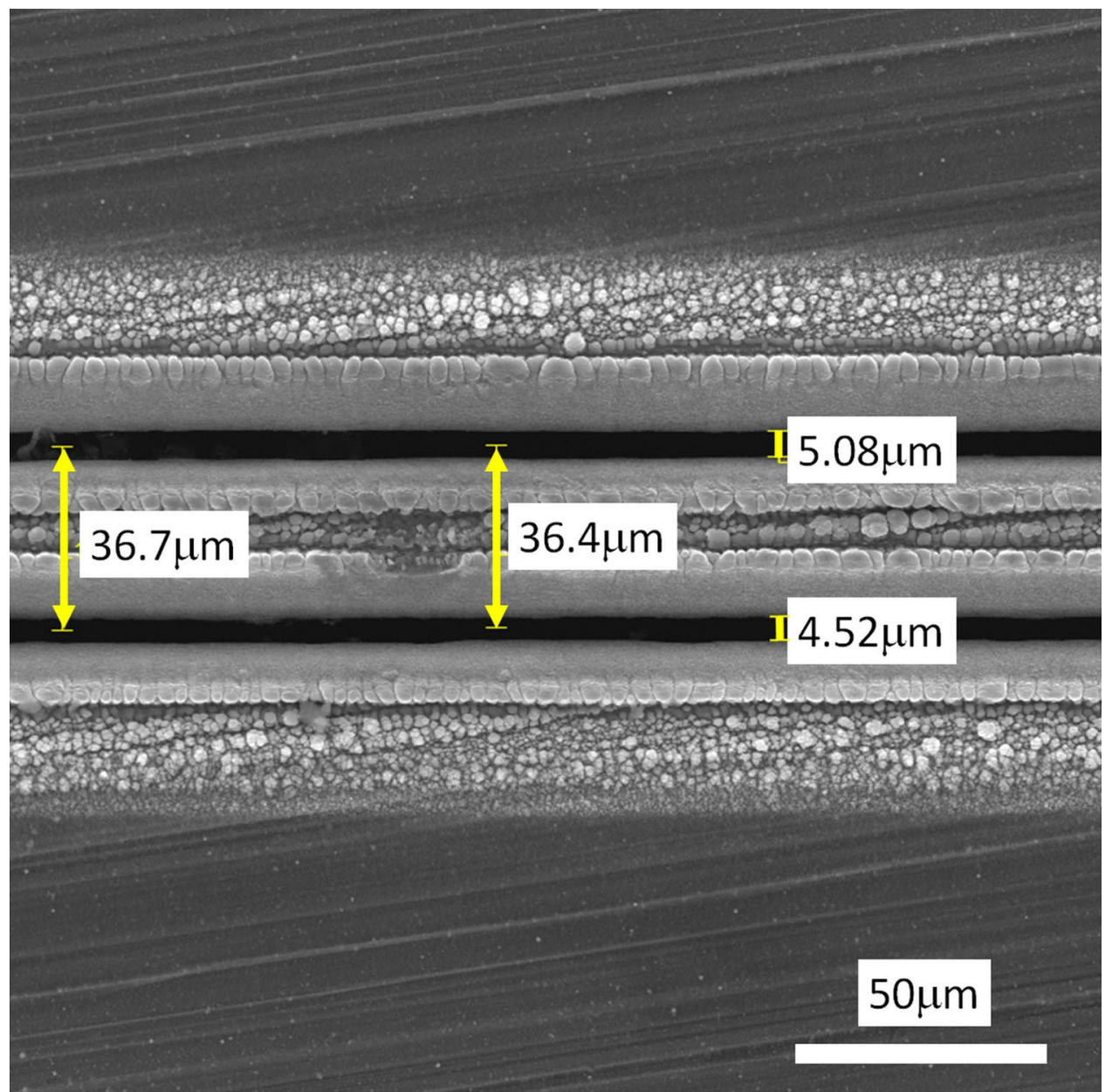

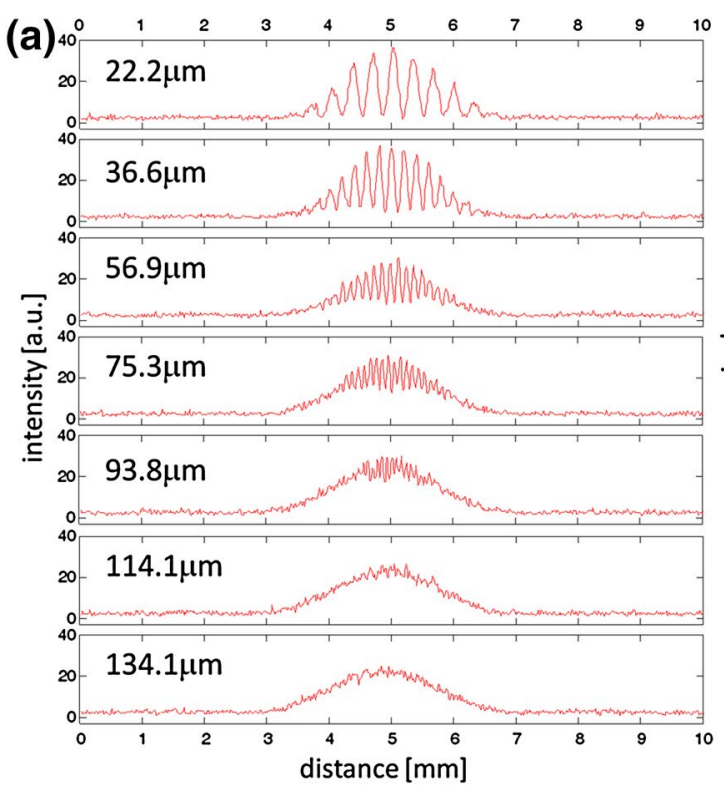

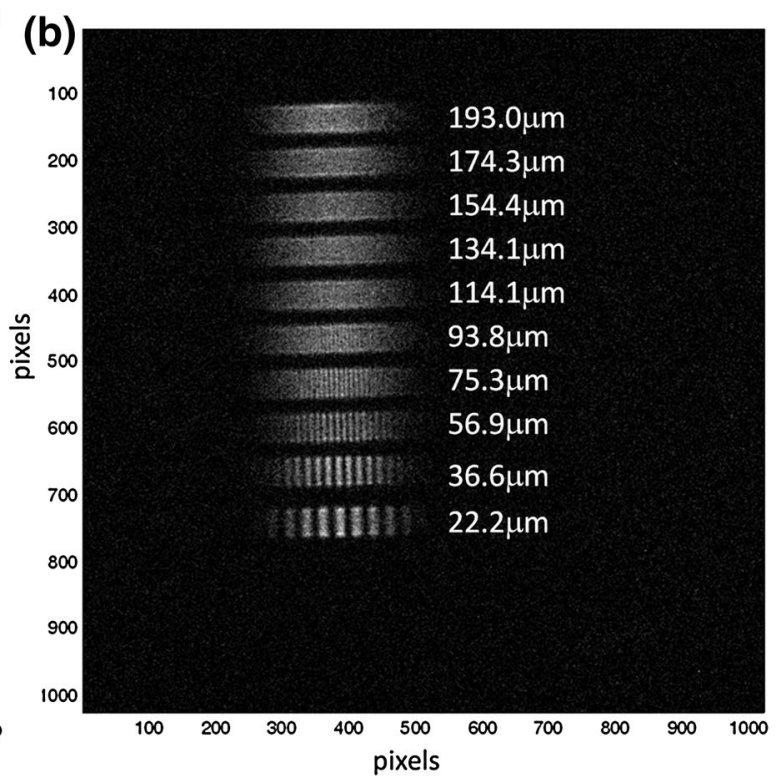

Fig. 3 Spatial coherence measurements in horizontal direction (a) modulation profiles obtained from the experimental data (b) for various slit separations indicated in the images 

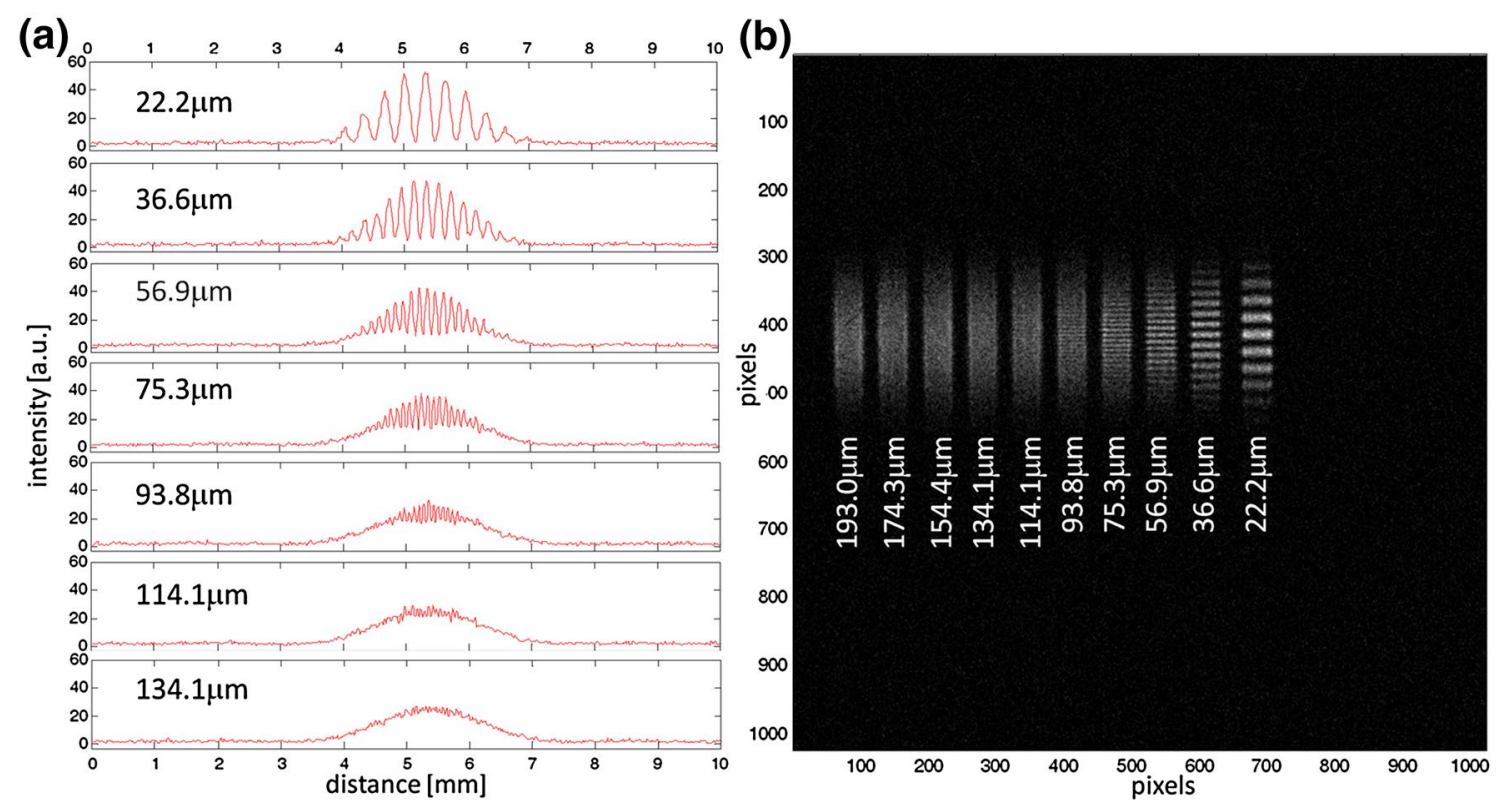

Fig. 4 Same measurements as in Fig. 2, except that performed in the vertical direction by rotating the arrangement with variable separation slits by $90^{\circ}$ in respect to the position from Fig. 2

Fig. 5 Visibility, or absolute value of complex coherence factor as a function of the slit separations for horizontal and vertical measurements. Inset shows the SEM image of the pinhole used for spatial filtering of the Xe plasma EUV radiation. Gaussian profiles with radius of coherence were adjusted to fit each set of data, resulting in 56 and $59 \mu \mathrm{m}$ radii of coherence for horizontal and vertical measurements performed at the distance of $2.1 \mathrm{~m}$ from the plasma

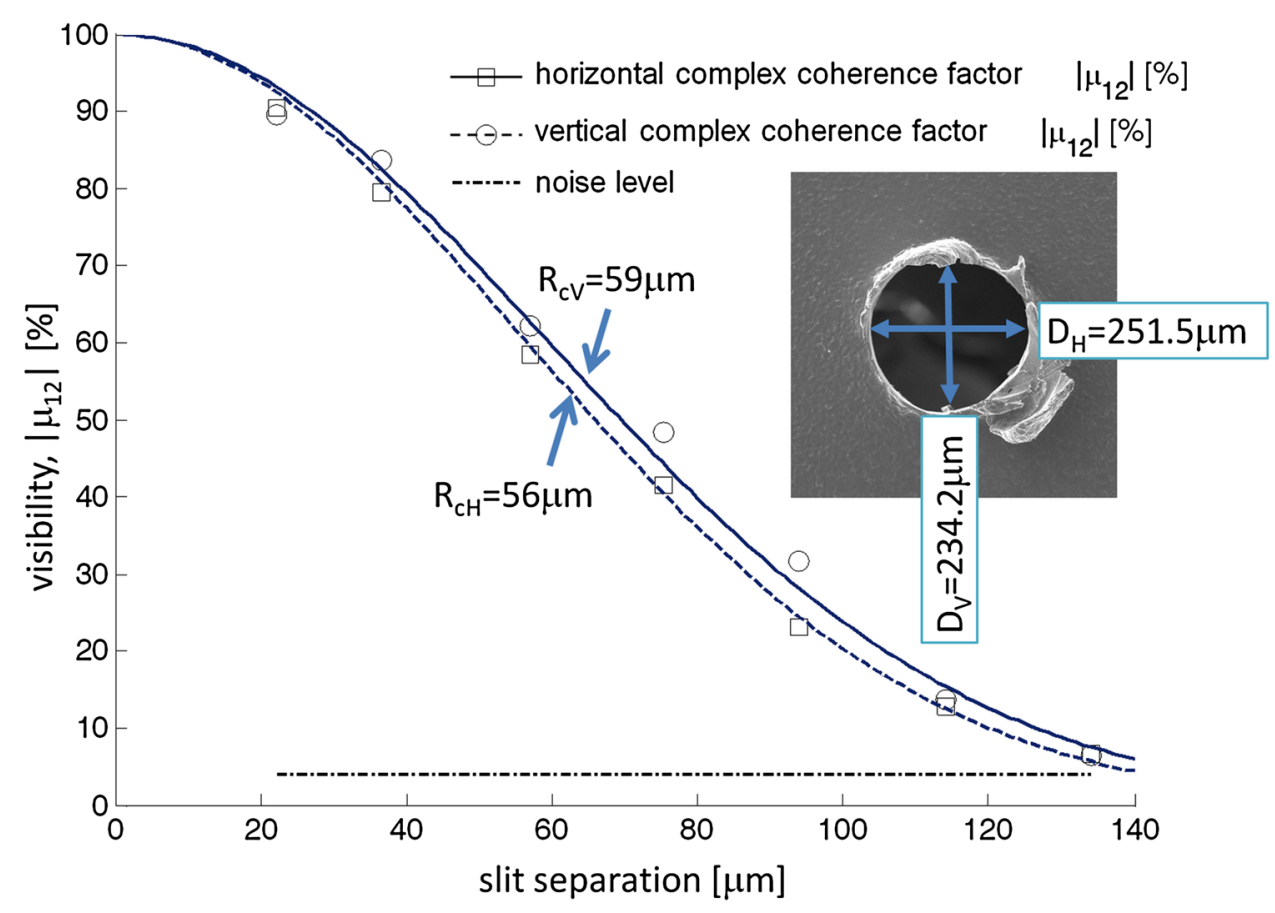

the pinhole size between $D_{\mathrm{H}}$ and $D_{\mathrm{V}}$ causes small changes in the interference pattern visibilities as well. It is difficult to see that immediately just from the intensity profiles in Figs. 3 and 4, however, can be better appreciated if the visibility versus the slit separation is plotted. This can be seen in Fig. 5, in which square markers indicate the visibility values obtained from the intensity profiles for horizontal measurements and circular markers for vertical measurements, respectively. In an ideal double slit experiment, in which both slits are uniformly illuminated, the modulus of the complex coherence factor is equal to the fringe visibility $\left|\mu_{12}\right|=V$ [23].

Thus, it is possible to directly derive information about the spatial coherence of the plasma emission by measurement of 
the fringe visibility. This was demonstrated in experiments by Thompson and Wolf with partially coherent visible light [24] and used multiple times to measure the spatial coherence, i.e., [20]. For the slit separation equal $\sim 134 \mu \mathrm{m}$, the modulation is still visible, so the visibility can be calculated; however, for higher slit separations, the visibility approaches $4 \%$, which is the limiting value here related to the CCD camera noise. Even from the marker locations, it is possible to see that the visibility drops slightly faster for in the case of horizontal measurements. It is in accordance with the theory, since $D_{\mathrm{H}}>D_{\mathrm{V}}$. It can be better appreciated if a Gaussian-type function is fitted to the data. This fitting has been performed and can be seen in Fig. 5, where the solid line represents the fitted curve for horizontal measurements and the dotted line for vertical measurements. In this work, we employed coherence radius $R_{\mathrm{C}}$ to characterize the spatial coherence parameter such as transversal coherence length. The $R_{\mathrm{C}}$ follows the convention of the area of coherence $A_{\mathrm{C}}$, which is often used in the literature [23, 25]. By definition

$A_{\mathrm{C}}=\pi R_{\mathrm{C}}^{2}=\iint\left|\mu_{12}(\Delta x, \Delta y)\right|^{2} \mathrm{~d} \Delta x \cdot \mathrm{d} \Delta y$

where $\mu_{12}(\Delta x, \Delta y)$ is the complex coherence factor. Therefore, a Gaussian profile to fit into the visibility data would be

$\left|\mu_{12}(\Delta x, \Delta y)\right|=\exp \left[\frac{-\left(\Delta x^{2}+\Delta y^{2}\right)}{2 R_{\mathrm{C}}^{2}}\right]=\exp \left(\frac{-\Delta r^{2}}{2 R_{\mathrm{C}}^{2}}\right)$

where $\Delta r$ is the distance between two point sources of electromagnetic waves producing the interference pattern; in our case in its discrete form, it is equivalent to slit separation. For the horizontal measurements, $\Delta y=0$ and $\left|\mu_{12}(\Delta x)\right|=\exp \left(-0.5 \Delta x^{2} / R_{\mathrm{cH}}^{2}\right)$ which was fitted to the horizontal data points (squares) resulting in radius of coherence in horizontal direction of $R_{\mathrm{cH}}=56 \mu \mathrm{m}$.

The same procedure was also performed in the orthogonal direction fitting $\left|\mu_{12}(\Delta y)\right|=\exp \left(-0.5 \Delta y^{2} / R_{\mathrm{cV}}^{2}\right)$ to the circular data points and obtaining $R_{\mathrm{cV}}=59 \mu \mathrm{m}$. Those measurements are in accordance with the source size enforced by a pinhole placed in front of it, since the ratio $R_{\mathrm{cV}} / R_{\mathrm{cH}}=1.054$ differs from $D_{\mathrm{H}} / D_{\mathrm{V}}=1.074$ by $1.8 \%$. Moreover, taking into account that

$D_{\mathrm{C}}=2 R_{\mathrm{C}} \sim \frac{\lambda z}{D}$

where $D_{\mathrm{C}}$ is the diameter of coherence, $z$ is the source (pinhole) to the mask (plane at which the coherence measurements have been performed) distance, and $D$ is the diameter of the source defined by the spatial filtering pinhole [26]. In addition, considering both orthogonal direction, at which the measurements were carried out, the equations are satisfied with a coupling factors of $2 R_{\mathrm{cH}}=0.994 \lambda z / D_{\mathrm{H}}$ and
$2 R_{\mathrm{cV}}=0.975 \lambda z / D_{\mathrm{V}}$. From that, also an effective incoherent source size (diameter) can be derived:

$d_{\mathrm{s}}=\frac{2 \lambda z}{\sqrt{\pi A_{\mathrm{C}}}}=\frac{2 \lambda z}{\pi R_{\mathrm{C}}}$

which in this case is equal to $d_{\mathrm{sH}}=322 \mu \mathrm{m}$ and $d_{\mathrm{sV}}=306 \mu \mathrm{m}$. This is the diameter of a uniform, incoherent source which yields the same coherence area as that measured.

\section{Application of partially coherent EUV radiation from xenon plasma to holography}

To apply those results to imaging a proof of principle, Gabor holography experiments was performed. The mask with slits was replaced by an object made of different diameter metal wires. The object was illuminated by a partially spatially coherent EUV beam with radius of coherence equal to $R_{\mathrm{C}}=60 \mu \mathrm{m}$, at the distance from the pinhole, which is approximately the same as the distance to the source equal to $z_{\mathrm{s}}=2.1 \mathrm{~m}$ and with an inverse spectral bandwidth $\lambda / \Delta \lambda=14$. The CCD camera was closer to the object than before, at the distance of $z_{\mathrm{p}}=22 \mathrm{~cm}$ from the object. With those parameters, the spatial resolution was limited theoretically to $6.2 \mu \mathrm{m}$ by the bandwidth of the EUV emission and to $30.2 \mu \mathrm{m}$ by the transversal coherence. The resolution can be further improved by reducing $z_{\mathrm{p}}$, until the resolution limit imposed by the CCD camera pixel size of $13 \times 13 \mu \mathrm{m}^{2}$. After the illumination, the part of the EUV beam scatters from the object (object beam) and interferes with an un-obscured beam (reference beam) propagating through the openings of the object, producing at the CCD plane an interference pattern. This pattern is a hologram. To obtain a single hologram, $30 \mathrm{EUV}$ pulses were used generated with $5 \mathrm{~J}$ energy laser pulses at $10 \mathrm{~Hz}$ repetition rate. Example of such hologram is depicted in Fig. 6a and zoomed in Fig. 6c, It depicts representation of the object- two crossed wires 150 and $20 \mu \mathrm{m}$ in diameter, Fig. $6 \mathrm{c}$. As can be seen, the wires' edges are surrounded by fringes obtained due to the interference between object and reference beams. Although the diameter of those wires differs by a factor of 7.5, both wires in the hologram appear to be quite similar in width. The only difference is their "intensity", as depicted in false colors in Fig. 6c. The hologram was numerically reconstructed with a Fresnel propagator [27] using approach similar to [28, 29]. A plane wave was back-propagated by the Fresnel Zone Plate of focal length given by

$\frac{1}{f}=\frac{1}{z_{\mathrm{p}}}-\frac{1}{z_{\mathrm{s}}+z_{\mathrm{p}}}$

and rescaled by the geometric magnification equal to $M=\left(z_{\mathrm{s}}+z_{\mathrm{p}}\right) / z_{\mathrm{s}}$ of the object as it is projected onto the CCD 


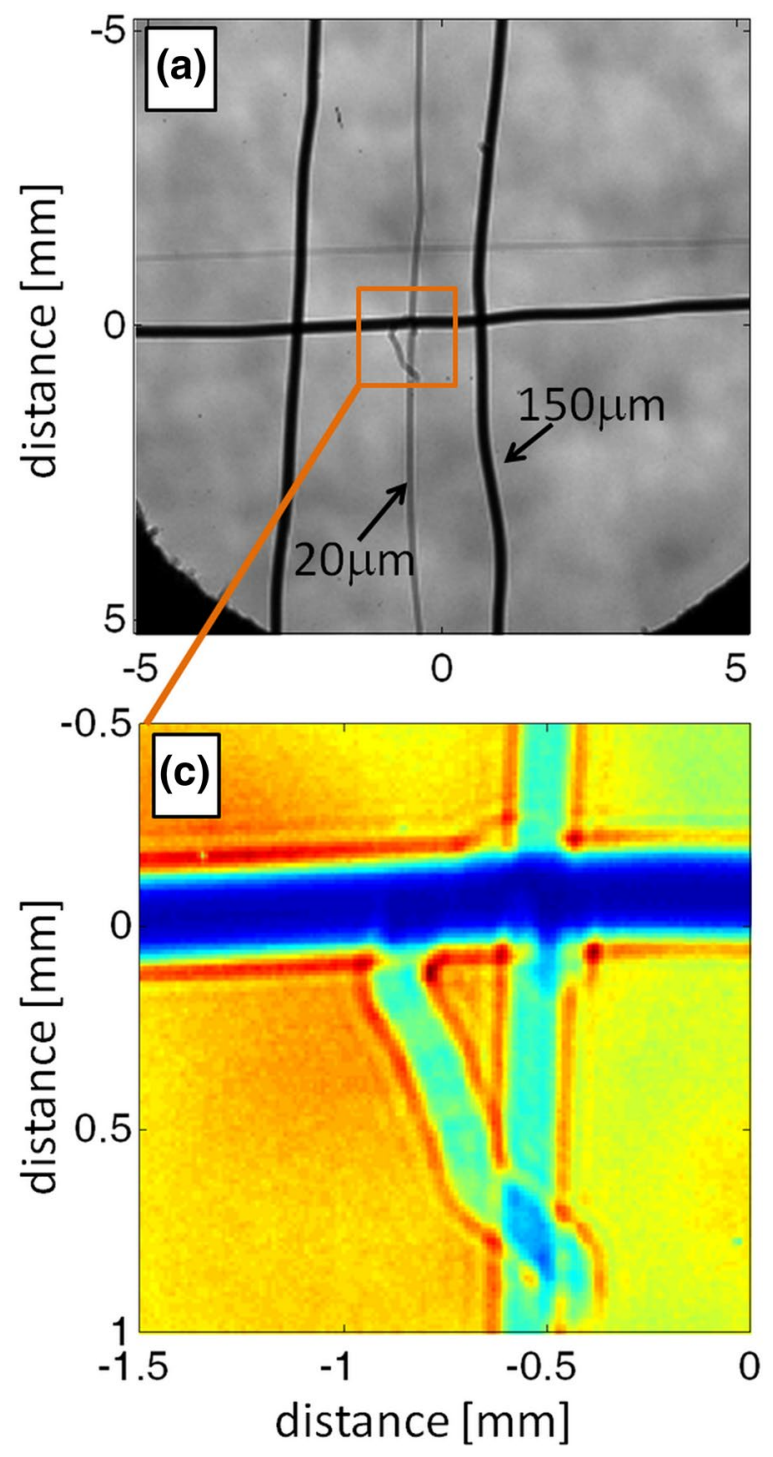

Fig. 6 Demonstration of the use of spatially coherent EUV emission from $\mathrm{Xe} / \mathrm{He}$ double stream gas puff target for Gabor holography. Holograms (a, c) and reconstructions (b, d) of an arrangement of two wires 150 and $20 \mu \mathrm{m}$ in diameter. Hologram shows wires almost the same thickness, just different intensities (c) (in false colors to indi-

camera. The propagation operation was done in the Fourier domain and back-transformed to the spatial domain through equation:

$$
\mathrm{R}(x, y)=\mathfrak{J}^{-1}\left\{H\left(f_{x}, f_{y}\right) \cdot F\left(f_{x}, f_{y}\right)\right\}
$$

where $\mathrm{R}(x, y)$ is the reconstructed image of the object in spatial domain, $H\left(f_{x}, f_{y}\right)$ is the hologram in the spatial frequency domain, $F\left(f_{x}, f_{y}\right)$ is the Fresnel zone plate propagator in the spatial frequency domain, and $\mathfrak{\Im}^{-1}$ denotes inverse 2D Fourier transform operation.

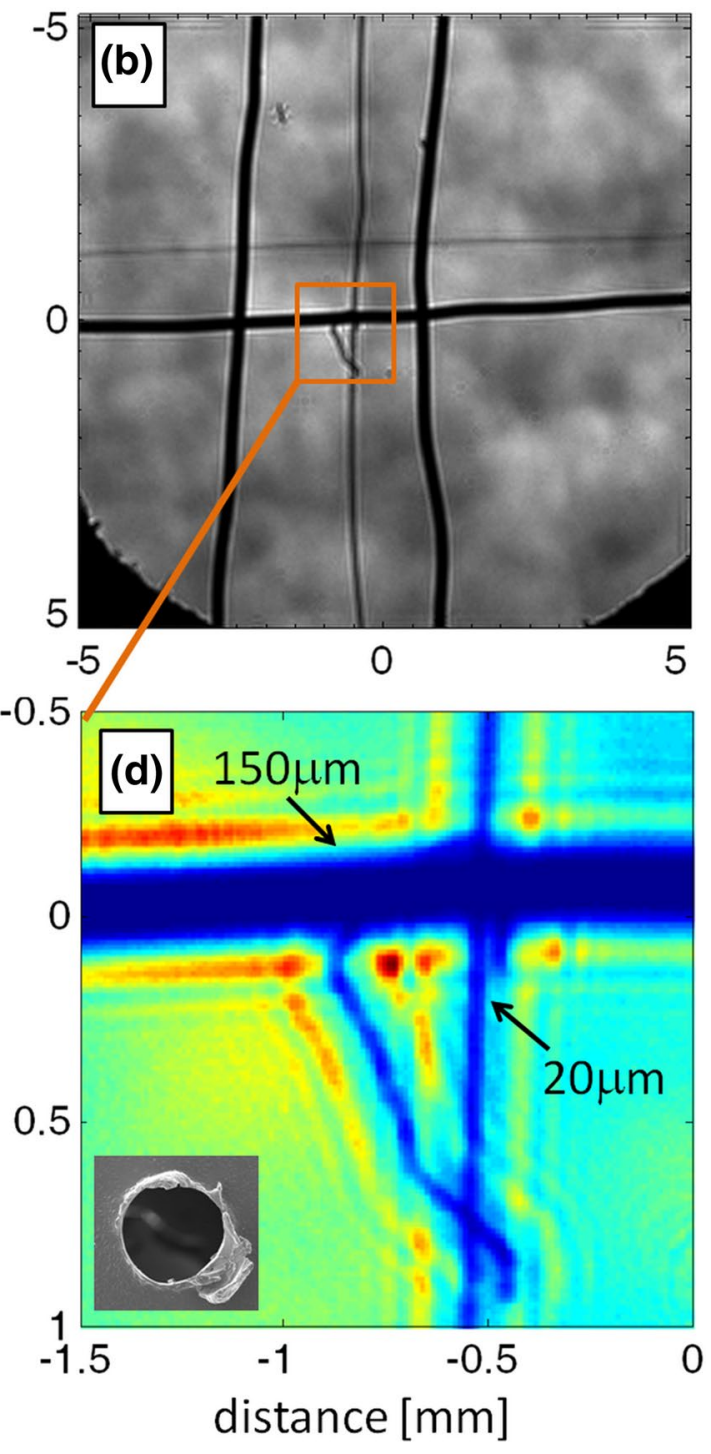

cate the variation in the intensity), while reconstruction by wavefront back-propagation (see text) makes possible to recover the original shape of the wire, which is much smaller than the source size indicated by the inset of the $0.25-\mathrm{mm}$ diameter source pinhole (d)

The reconstructed image of the wire objects is depicted in Fig. 6b. The zoomed image in Fig. 6d indicates the smaller wire reconstructed properly through the back-propagation having the size of $\sim 27 \mu \mathrm{m}$ (two CCD pixels), so taking into account the magnification factor of 1.1, the diameter of the wire is equal to $24.4 \mu \mathrm{m}$, in good agreement with the real value of $20 \mu \mathrm{m}$.

In addition, after the reconstruction, the two wires, being in fact completely opaque at $13.5-\mathrm{nm}$ wavelength, appear, as expected, to have similar "intensity". The small inset in Fig. $6 \mathrm{~d}$ shows also the pinhole used for spatial filtering of the EUV radiation in a proper scale. The figure shows that 
the diameter of the $20-\mu \mathrm{m}$ wire is much smaller than the diameter of the pinhole. Therefore, the reconstructed image has nothing to do with a simple shadowing effect introduced by the pinhole, limiting resolution in classical radiography or shadowgraphy.

It is also possible to roughly estimate the number of photons that took part in forming the hologram image by investigating the number of CCD counts in the pixels of a chip. On average, the number of counts (excluding the CCD background) per CCD pixel is 5218. Taking into account the quantum efficiency of the CCD camera chip at $13.5-\mathrm{nm}$ wavelength of $90 \%$ and considering that one EUV photon produces statistically $\sim 25.2$ photoelectrons, one can recalculate it to the statistical number of photons, obtaining 7.5 [photons/pixel/EUV pulse]. Taking into account the magnification of the system $(1.1 \times)$, this corresponds to $5.4 \times 10^{4}$ [photons $/ \mathrm{mm}^{2} / \mathrm{EUV}$ pulse] at the object plane. In the almost fully coherent case for slit separation of $\sim 22 \mu \mathrm{m}$, for which the $\left|\mu_{12}(\Delta r)\right|=90 \%$, there is $\sim 20$ [photons/EUV pulse] in the circular area with the diameter equal to that separation at $2.1 \mathrm{~m}$ away from the plasma source. Moreover, the circular area with radius equal to $R_{\mathrm{C}}=60 \mu \mathrm{m}$ contains $\sim 150$ [photons/EUV pulse] also at $2.1 \mathrm{~m}$ away from the plasma source.

\section{Conclusions and discussion}

In the presented work, spatial coherence measurements on the EUV emission from xenon plasma, formed by interaction of nanosecond laser pulses with a double stream gas puff target, are presented. The measurements show the change of the spatial coherence depending on the source size, which could be measured due to the asymmetry of the spatial filter. The measured radius of coherence was approximately equal to $R_{\mathrm{C}}=60 \mu \mathrm{m}$.

Due to the discrepancy between the slit widths, as can be seen in Fig. 2, of the order of 10\% for min-max deviation of the slit widths, the visibility measurements are affected by this fact. The visibility is the highest if two interfering beams have equal intensities. In this case, due to a small difference of the slit widths, the intensities of two interfering beams are differ also by a factor of $10 \%$, which theoretically leads to the visibility reduction of $0.14 \%$.

Additional effect, which influences the measurements, is the fact that the complex coherence factor $\mu_{12}$ can be used to measure the spatial coherence, assuming that the light is quasi-monochromatic, so the influence of temporal coherence is not present; however, in our case, $\Delta \lambda / \lambda=0.07$, so the non-zero bandwidth may influence the results as well. To calculate if this is the case, let us consider that coherence length of such Xe plasma source is equal to $l_{\mathrm{c}}=\lambda^{2} / \Delta \lambda=189 \mathrm{~nm}$. The condition for quasi-monochromaticity and application of $\mu_{12}=\gamma_{12}(0)$ instead of complex degree of coherence $\gamma_{12}(\tau)$, [30] can be only done if

$O P D=\left(r_{2}-r_{2}^{\prime}\right)-\left(r_{1}-r_{1}^{\prime}\right)<<l_{c}$

where $O P D$ is the optical path difference, $r_{1}$ and $r_{2}$ are the distances from any point of the emitting source to the two slits, and $r_{1}^{\prime}$ and $r_{2}^{\prime}$ are the distances from each slit to the detector. In our case, for the full range of slit separations, from $\sim 22.2$ to $193 \mu \mathrm{m}$, the OPD is equal to $1.3-11.5 \mathrm{~nm}$, respectively, from 16 to 143 times less than the coherence length of the source. Thus, the quasi-monochromaticity condition was satisfied in this case and the use of $\mu_{12}$ is justified. In conclusion, the xenon laser plasma source based on a double stream gas puff target, which is intrinsically incoherent, can in fact be modified to obtain partial spatial coherence, which was estimated, with the number of coherently emitted photons sufficient to perform coherent-type imaging, such as holography in Gabor on-axis configuration.

Acknowledgements This work is supported by the National Science Centre, Opus programme, grant agreement number UMO-2015/17/B/ ST7/03718 and UMO-2015/19/B/ST3/00435 and from the European Union's Horizon 2020 research and innovation program, under Laserlab-Europe IV, Grant agreement No. 654148.

Open Access This article is distributed under the terms of the Creative Commons Attribution 4.0 International License (http:// creativecommons.org/licenses/by/4.0/), which permits unrestricted use, distribution, and reproduction in any medium, provided you give appropriate credit to the original author(s) and the source, provide a link to the Creative Commons license, and indicate if changes were made.

\section{References}

1. H.N. Chapman, P. Fromme, A. Barty, T.A. White, R.A. Kirian, A. Aquila, M.S. Hunter, J. Schulz, D.P. DePonte, U. Weierstall, R.B. Doak, F.R.N.C. Maia, A.V. Martin, I. Schlichting, L. Lomb, N. Coppola, R.L. Shoeman, S.W. Epp, R. Hartmann, D. Rolles, A. Rudenko, L. Foucar, N. Kimmel, G. Weidenspointner, P. Holl, M. Liang, M. Barthelmess, C. Caleman, S. Boutet, M.J. Bogan, J. Krzywinski, C. Bostedt, S. Bajt, L. Gumprecht, B. Rudek, B. Erk, C. Schmidt, A. Hömke, C. Reich, D. Pietschner, L. Strüder, G. Hauser, H. Gorke, J. Ullrich, S. Herrmann, G. Schaller, F. Schopper, H. Soltau, K. Kühnel, M. Messerschmidt, J.D. Bozek, S.P. Hau-Riege, M. Frank, C.Y. Hampton, R.G. Sierra, D. Starodub, G.J. Williams, J. Hajdu, N. Timneanu, M.M. Seibert, J. Andreasson, A. Rocker, O. Jönsson, M. Svenda, S. Stern, K. Nass, R. Andritschke, C. Schröter, F. Krasniqi, M. Bott, K.E. Schmidt, X. Wang, I. Grotjohann, J.M. Holton, T.R.M. Barends, R. Neutze, S. Marchesini, R. Fromme, S. Schorb, D. Rupp, M. Adolph, T. Gorkhover, I. Andersson, H. Hirsemann, G. Potdevin, H. Graafsma, B. Nilsson, J.C.H. Spence, Femtosecond X-ray protein nanocrystallography. Nature 470(7332), 73-77 (2011)

2. Y. Nishino, Y. Tanaka, M. Okada, M. Okaya, Y. Uozaki, K. Nozaki, M. Yabashi, M. Nagasono, K. Tono, H. Kimura, Femtosecond snapshot holography with extended reference using extreme ultraviolet free-electron laser. Appl. Phys. Express 3, 10 (2010) 
3. OYu. Gorobtsov, G. Mercurio, G. Brenner, U. Lorenz, N. Gerasimova, R.P. Kurta, F. Hieke, P. Skopintsev, I. Zaluzhnyy, S. Lazarev, D. Dzhigaev, M. Rose, A. Singer, W. Wurth, I.A. Vartanyants, Statistical properties of a free-electron laser revealed by Hanbury Brown-Twiss interferometry. Phys. Rev. A 95, 023843 (2017)

4. S. Heinbuch, M. Grisham, D. Martz, J.J. Rocca, Demonstration of a desk-top size high repetition rate soft x-ray laser. Opt. Express 13(11), 4050-4055 (2005). doi:10.1364/OPEX.13.004050

5. T.T. Luu, H.J. Wörner, High-order harmonic generation in solids: a unifying approach. Phys. Rev. B 94, 115164 (2016)

6. B.A. Reagan, M. Berrill, K.A. Wernsing, C. Baumgarten, M. Woolston, J.J. Rocca, High-average-power, 100-Hz-repetitionrate, tabletop soft-x-ray lasers at sub-15-nm wavelengths. Phys. Rev. A 89, 053820 (2014). doi:10.1103/PhysRevA.89.053820

7. M.D. Seaberg, D.E. Adams, E.L. Townsend, D.A. Raymondson, W.F. Schlotter, Y. Liu, C.S. Menoni, L. Rong, C. Chen, J. Miao, H.C. Kapteyn, M.M. Murnane, Ultrahigh $22 \mathrm{~nm}$ resolution coherent diffractive imaging using a desktop $13 \mathrm{~nm}$ high harmonic source. Opt. Express 19(23), 22470 (2011)

8. P.W. Wachulak, M.C. Marconi, R. Bartels, C.S. Menoni, J.J. Rocca, Soft X-ray holography with wavelength resolution. J. Opt. Soc. Am. B 25(11), 1811-1814 (2008). doi:10.1364/ JOSAB.25.001811

9. G. D. Kubiak, L. J. Bernardez, K. D. Krenz, High-power extreme-ultraviolet source based on gas jets. In: Proceedings of SPIE 3331, Emerging Lithographic Technologies II, 81 (1998), doi: $10.1117 / 12.309560$

10. H. Fiedorowicz, A. Bartnik, R. Jarocki, J. Kostecki, J. Krzywiński, J. Mikołajczyk, R. Rakowski, A. Szczurek, M. Szczurek, Compact laser plasma EUV source based on a gas puff target for metrology applications. J. Alloy. Compd. 401(1-2), 99-103 (2005). doi:10.1016/j.jallcom.2005.02.069

11. P.W. Wachulak, A. Torrisi, A. Bartnik, D. Adjei, J. Kostecki, L. Wegrzynski, R. Jarocki, M. Szczurek, H. Fiedorowicz, Desktop water window microscope using a double-stream gas puff target source. Appl. Phys. B 118(4), 573-578 (2015). doi:10.1007/ s00340-015-6044-x

12. A. Bartnik, R. Fedosejevs, P. Wachulak, H. Fiedorowicz, C. Serbanescu, E.G. Saiz, D. Riley, S. Toleikis, D. Neely, Photo-ionized neon plasmas induced by radiation pulses of a laser-plasma EUV source and a free electron laser FLASH. Laser Part. Beams 31(2), 195-201 (2013)

13. A. Bartnik, H. Fiedorowicz, R. Jarocki, J. Kostecki, M. Szczurek, A. Biliński, O. Chernyayeva, J.W. Sobczak, EUV-induced surface modification of polymers, in X-ray lasers 2010 , vol. 136, Springer Proceedings in Physics, ed. by J. Lee, C.H. Nam, K.A. Janulewicz (Springer, Dordrecht, 2011)

14. D. Adjei, M. Getachew Ayele, P. Wachulak, A. Bartnik, Ł. Wegrzynski, H. Fiedorowicz, L. Vyšín, A. Wiechec, J. Lekki, W.M. Kwiatek, L. Pina, M. Davídková, L. Juha, Development of a compact laser-produced plasma soft X-ray source for radiobiology experiments. Nucl. Instrum. Methods Phys. Res. Sect. B 364, 27-32 (2015). doi:10.1016/j.nimb.2015.08.065
15. P.W. Wachulak, A. Bartnik, H. Fiedorowicz, T. Feigl, R. Jarocki, J. Kostecki, R. Rakowski, P. Rudawski, M. Sawicka, M. Szczurek, A. Szczurek, Z. Zawadzki, A compact, quasi-monochromatic laser-plasma EUV source based on a double-stream gas-puff target at $13.8 \mathrm{~nm}$ wavelength. Appl. Phys. B 100(3), 461-469 (2010)

16. R. Rakowski, A. Bartnik, H. Fiedorowicz, F. de Gaufridy, R. de Dortan, J. Jarocki, J. Kostecki, L. Mikołajczyk, M. Ryć, P.Wachulak Szczurek, Characterization and optimization of the laser-produced plasma EUV source at $13.5 \mathrm{~nm}$ based on a doublestream Xe/He gas puff target. Appl. Phys. B 101(4), 773-789 (2010)

17. P.W. Wachulak, A. Bartnik, M. Skorupka, J. Kostecki, R. Jarocki, M. Szczurek, L. Wegrzynski, T. Fok, H. Fiedorowicz, Waterwindow microscopy using a compact, laser-plasma SXR source based on a double-stream gas-puff target. Appl. Phys. B 111(2), 239-247 (2013)

18. D.T. Attwood, Soft X-rays and Extreme Ultraviolet Radiation (Cambridge University Press, New York, 1999)

19. P. Lu, E. Fill, Y. Li, J. Maruhn, G. Pretzler, Spatial coherence of prepulse-induced neonlike x-ray lasers. Phys. Rev. A 58, 628 (1998)

20. T. Ditmire, E.T. Gumbrell, R.A. Smith, J.W.G. Tisch, D.D. Meyerhofer, M.H.R. Hutchinson, Spatial coherence measurement of soft X-ray radiation produced by high order harmonic generation. Phys. Rev. Lett. 77, 4756 (1996)

21. Y. Liu, M. Seminario, F.G. Tomasel, C. Chang, J.J. Rocca, D.T. Attwood, Achievement of essentially full spatial coherence in a high-average-power soft-x-ray laser. Phys. Rev. A 63, 033802 (2001). doi:10.1103/PhysRevA.63.033802

22. M. Born, E. Wolf, Principles of Optics, 7th edn. (Cambridge University Press, Cambridge, 1999), p. 572

23. J.W. Goodman, Statistical Optics (Wiley, New York, 1985), pp. 171-187

24. B.J. Thompson, E. Wolf, Two-beam interference with partially coherent light. J. Opt. Soc. Am. 47, 895 (1957)

25. M. Born, E. Wolf, Principles of Optics, 7 th edn. (Cambridge University Press, Cambridge, 1999), p. 576

26. Ian Mc Nulty, Coherence and partial coherence - what do we need?, MBA Lattice Workshop, Advanced Photon Source 21-22 October 2013, Argonne National Laboratory

27. J.W. Goodman, Introduction to Fourier Optics (Roberts and Company Publishers, Greenwood Village, 2005)

28. R.A. Bartels, A. Paul, H. Green, H.C. Kapteyn, M.M. Murnane, S. Backus, I.P. Christov, Y. Liu, D. Attwood, C. Jacobsen, Generation of spatially coherent light at extreme ultraviolet wavelengths. Science 297, 376-378 (2002)

29. P.W. Wachulak, R.A. Bartels, M.C. Marconi, C.S. Menoni, J.J. Rocca, Y. Lu, B. Parkinson, Sub $400 \mathrm{~nm}$ spatial resolution extreme ultraviolet holography with a table top laser. Opt. Express 14(21), 9636 (2006)

30. J.W. Goodman, Statistical Optics, polish edn. (Wydawnictwo Naukowe PWN, Warsaw, 1993), p. 168 